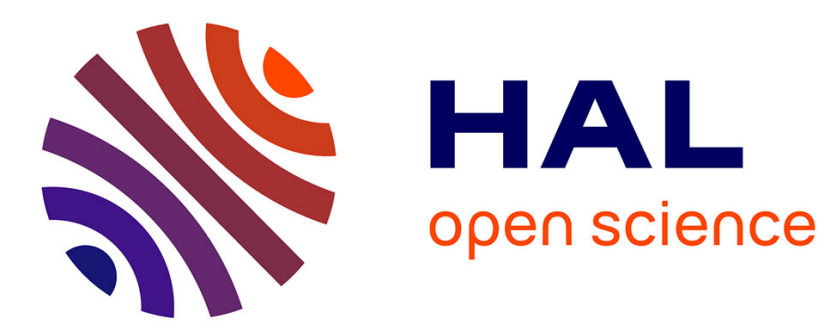

\title{
Model correlation and identification of experimental reduced models in vibroacoustical modal analysis
}

Morvan Ouisse, Emmanuel Foltete

\section{To cite this version:}

Morvan Ouisse, Emmanuel Foltete. Model correlation and identification of experimental reduced models in vibroacoustical modal analysis. Journal of Sound and Vibration, 2015, 342, pp.200 - 217. hal-02131273

\section{HAL Id: hal-02131273 \\ https://hal.science/hal-02131273}

Submitted on 16 May 2019

HAL is a multi-disciplinary open access archive for the deposit and dissemination of scientific research documents, whether they are published or not. The documents may come from teaching and research institutions in France or abroad, or from public or private research centers.
L'archive ouverte pluridisciplinaire HAL, est destinée au dépôt et à la diffusion de documents scientifiques de niveau recherche, publiés ou non, émanant des établissements d'enseignement et de recherche français ou étrangers, des laboratoires publics ou privés. 
This document is the author's final manuscript of

M. Ouisse, E. Foltête: Model correlation and identification of experimental reduced models in vibroacoustical modal analysis. Journal of Sound and Vibration 342 (2015) 200-217.

This paper has been published by Elsevier and can be found at http://dx.doi.org/10.1016/j.jsv.2014.12.042 


\title{
Model correlation and identification of experimental reduced models in vibroacoustical modal analysis
}

\author{
Morvan OUISSE \\ FEMTO-ST Applied Mechanics - 24, chemin de l'épitaphe - 25000 Besançon - FRANCE
}

Emmanuel FOLTÊTE

\begin{abstract}
An important issue in vibroacoustics is the topic of verification and validation of models for design. This paper addresses several points in this context: model correlation, optimal test design and identification of reduced models. Model correlation criteria like Modal Assurance Criteria (MAC) are widely used in structural dynamics in order to check the spatial coincidence between two sets of vectors. This concept is extended here to vibroacoustic couplings, together with optimal test design strategies: on the basis of a given model, they allow finding of the best sensors and actuators placements for the modal analysis. Finally, the last part of the paper proposes a methodology allowing identification of experimental reduced models from the knowledge of the complex modes issued from the vibroacoustical modal analysis. Both numerical and experimental results are used to check the efficiency of the procedure.
\end{abstract}

\section{Introduction}

Vibroacoustics constitute an engineering area in which multiphysic analyses are of first importance because of the couplings between structural vibrations and acoustic waves in air. We focus here on cases where the acoustic domain is a cavity, surrounded by an elastic domain, which is representative of many practical situations in the fields of transports, buildings, etc. A lot of efforts have been devoted to the development of numerical models to deal with this kind of problem using finite elements models $[1,2,3]$. This approach is known to be efficient in the low frequency domain, and this paper will not address the situations in which modal overlap becomes large enough to consider alternatives to the finite elements technique [4]. In the model-based design context, model validation is a central point that constitutes a research topic of growing interest [5]. The most popular way consists in validating conservative models using correlation and model updating on normal modes, even if frequency response functions may also be used [6], in particular for damping identification [7]. Some authors also investigated the possibility to use time responses for validation [8]. It should be emphasized that very few papers are available in literature concerning model validation for vibroacoustics. The reference paper

Email addresses: morvan. ouisse@univ-f comte.fr (Morvan OUISSE), emmanuel.foltete@univ-fcomte.fr (Emmanuel FOLTÊTE) 
by Wyckaert et al. [9] extends the concept of modal analysis to structural-acoustics coupling and is considered as an important input to the work presented in this paper.

In many engineering applications, the main goal is the reduction of acoustic and vibration levels, and damping devices are key points for effective design. An important issue is clearly the ability to correctly handle viscoelastic $[10,11]$ or poroelastic $[12,13]$ materials, even if this implies often a large increase in terms of model parameters, which is not always easy to deal with [14]. In this context, the identification of analytical models which are able to properly represent the behavior of the system is still an open question, in particular concerning the damping terms. Both stiffness and mass can be derived quite easily from models, or even from experiments with reasonable confidence. As far as the dissipative effects are concerned, there is still no consensus about the most reliable technique to obtain a physical description of damping which can be efficient for simulation. Damping aspects have been at the center of many works, among which the famous papers from Caughey [15] including considerations about normal and complex modes, which are of first importance in the context of interest. Some review papers have been published $[16,17,18]$, including many references to important works on damping related aspects. One of the ways to identify damping consists in finding a reduced model directly from experimental data. In this paper we will mainly focus on this kind of approach, which allows the identification of second-order matrices corresponding to classical stiffness, mass and viscous damping terms of multi-degrees of freedom models. This topic has shown a growing interest over the last decades in structural dynamics, but almost no comparable work can be found in open literature on vibroacoustics. It can be seen as a complementary approach to the reduced order techniques which are popular in numerical analyses: a lot of research has been devoted to the development of specific techniques for the derivation of numerical reduced models, based on various strategies among which bases enrichment or projection on complex modes [19, 20, 21, 22]. They are widely used for simulation, in particular in optimization runs and robustness analyses, that both require high number of calculations. They are usually derived from large numerical models and are useful to estimate physical quantities of interest. The alternative approach which is considered here, consists in using experimental data, and related works in literature can be basically classified in two categories. These categories are related to the nature of the input data: direct use of Frequency Response Functions (FRFs)(which are basically very efficient if excitation on all dofs are possible) [23], or use of complex modes, identified from the FRFs. A combination of both is also considered by some authors [24, 25]. Among these methods, the ones which are based on the use of a full modal basis (ie with a number of identified modes which is equal to the number of degrees of freedom in the reduced model) are the most efficient for correct damping localization. In these papers, the authors exhibit a large set of available methods, starting either from FRFs or modal data to identify at least the damping matrix. These methods are applied and compared on given test-cases. An interesting point is that these papers do not lead to the same conclusions concerning the efficiency of the techniques for practical applications, which clearly means that there is still some work to do.

This paper will be limited to the case where system matrices are obtained from identified complex modes, with a particular focus on the so-called properness condition. This condition, which is not addressed in the review papers referenced above, has been mentioned with various names in several publications [26, 27, 28, 29]. It is automatically verified by the exact complex modes of the system. When a full basis is extracted from experimental data to reconstruct a physical model, this condition should be enforced on the complex modes to obtain physical 
results. Balmès [30] has proposed a methodology to find optimal complex vectors which are as close as possible to the initial identified vectors, while verifying the so-called properness condition. This technique has been extended recently to non self-adjoint problems [31]. However it is not applicable for vibroacoustics because of the relationship between cross terms as it will be detailed in this paper. Another way to obtain optimal complex vectors from measured ones has been proposed by Adhikari [32,33], but this method requires the knowledge of real modes, which can be quite problematic in cases where damping has a great impact on structural behavior $[34,30]$.

The choice of the degrees of freedom which are used to identify the reduced model is an important point. A non-validated model may be efficiently used to determine their locations. This requires dedicated tools, namely model correlation and optimal test design strategies, which are proposed in the first parts of this paper. As explained in the text, they are inspired from existing techniques in structural dynamics.

This paper is organized as follows. Part 2 recalls the formulation of a vibroacoustic problem. Damping considerations are given and the expressions of complex modes for vibroacoustics are recalled, with a focus on the relationship between left and right eigenvectors. Some original comments about the validity of this relationship are given at the end of the part. Part 3 is dedicated to the definition of a correlation coefficient that can be used to correlate two models. Part 4 introduces a new technique for optimal correction of complex modes to estimate vibroacoustic experimental reduced model, which is validated through the use of a simple numerical test-case. Part 5 is dedicated to experimental validation of the approach, and includes a dedicated optimal test design approach. Finally, conclusions and perspectives are given at the end of the paper.

\section{Formulation of structural-acoustic problem}

This first part recalls the formulation which is used in this work for internal damped vibroacoustic problems.

\subsection{Coupled formulation}

The internal vibroacoustic problem which is considered in this paper is presented in figure 1 . The basic equations of the coupled problem are classically available in literature $[1,2,3]$. Let $\Omega_{F}$ be the fluid domain, $\Omega_{S}$ the structural domain. The boundary of the fluid domain is $\Sigma_{F S} \cup \Sigma_{F} \cup \Sigma_{A}$, where $\Sigma_{F S} \cap \Sigma_{F} \cap \Sigma_{A}=\emptyset$, while the boundary of the structural domain is $\Sigma_{F S} \cup \Sigma_{S} \cup \Sigma_{0}$, where $\Sigma_{F S} \cap \Sigma_{S} \cap \Sigma_{0}=\emptyset$. The partition of boundaries is done according to the mechanical conditions:

- $\Sigma_{F S}$ is the structural-acoustic coupling surface,

- $\Sigma_{F}$ is the part of the acoustic border on which a Neumann condition is applied, corresponding to a rigid wall (a homogeneous Dirichlet condition could also be considered without loss of generality),

- $\Sigma_{A}$ is the part of the acoustic border on which a Robin condition is considered, corresponding typically to an absorbing material,

- $\Sigma_{S}$ is the structural boundary on which a Neumann condition is applied, corresponding to a prescribed force, 


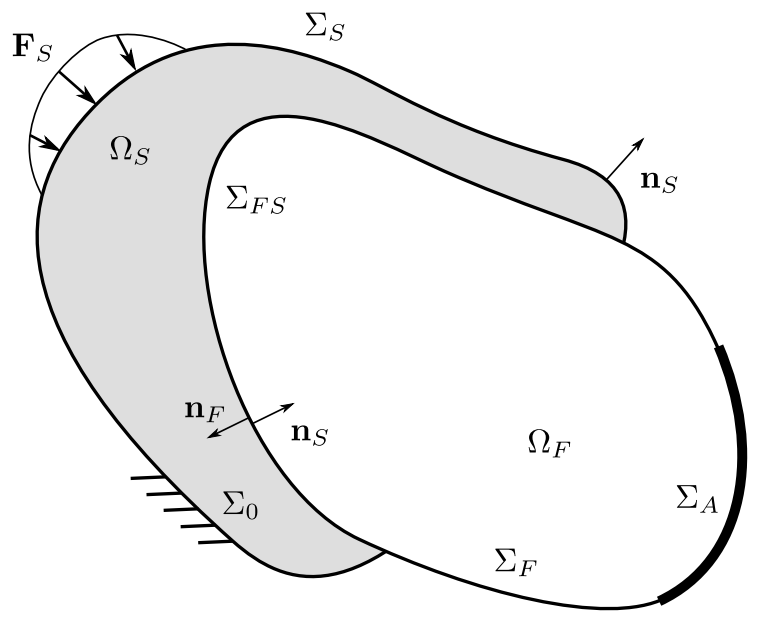

Figure 1: Description of vibroacoustic problem

- $\Sigma_{0}$ is the structural boundary on which a homogeneous Dirichlet condition is applied, corresponding to a clamped area.

Physical quantities of interest are the structural displacement field $\mathbf{u}$ and the acoustic pressure $p$ in the fluid domain. An algebraic model of the vibroacoustic system can be derived from a finite element discretization of the weak formulation associated to the local equations. For an harmonic excitation at frequency $\omega$, this typically yields to a problem such as

$$
(\underbrace{\left[\begin{array}{cc}
K_{S} & -L \\
0 & K_{F}
\end{array}\right]}_{[K]}+i \omega\left[\begin{array}{cc}
C_{S} & 0 \\
0 & 0
\end{array}\right]-\omega^{2} \underbrace{\left[\begin{array}{cc}
M_{S} & 0 \\
L^{T} & M_{F}
\end{array}\right]}_{[M]}+\frac{i \omega}{Z_{a}(\omega)}\left[\begin{array}{cc}
0 & 0 \\
0 & A_{F}
\end{array}\right]) \underbrace{\left\{\begin{array}{c}
U \\
P
\end{array}\right\}}_{\{q\}}=\underbrace{\left\{\begin{array}{c}
F_{S} \\
\dot{Q}
\end{array}\right\}}_{\{f\}},
$$

where the matrices are obtained by discretization of the integrals of the weak formulation using $N_{S}$ structural dofs $\{U\}$ associated to $\mathbf{u}$ and $N_{F}$ acoustic pressure dofs $\{P\}$ associated to $p$. $K_{S}$, $C_{S}$ and $M_{S}$ are respectively structural stiffness, damping and mass matrices, $K_{F}$ and $M_{F}$ are respectively named "stiffness" and "mass" matrices of fluid in the following. $L$ is the coupling matrix; $A_{F}$ is the matrix corresponding to the location of $\Sigma_{A} ; F_{S}$ is the external force vector acting on the structure, $\dot{Q}$ is the "acoustic force", which can be expressed in term of an acoustic volume acceleration of an ideal monopole [9]; $Z_{a}(\omega)$ is the acoustic impedance of the absorbing material used in the cavity.

\subsection{Damping considerations, complex modes}

In the context of low frequency applications for which modal analysis is one way to solve the problem, the difficult point comes from the frequency dependency of the acoustic impedance $Z_{a}$. In this work we propose a methodology which aims at finding an equivalent viscous matrix, representing the damping effects in the fluid domain. This damping effect is local by nature (corresponding to viscous and thermal effects in the porous layer), but since in the low frequency domain the damping effects mainly affect the response at resonance, various representations may 
reproduce similar global behaviors. In cases where the effect of the frequency-dependency of $Z_{a}$ on the global behavior in the frequency range of interest is such that the equivalent viscous model is no longer representative of the damping effects, it may be efficient to divide the frequency band in smaller bands in which the procedures can be applied [30]. It should be emphasized that similar comments may be done on the structural side where viscoelastic devices would lead to frequency-dependent terms in damping effects. If losses can be considered through the use of equivalent viscous damping terms in both structural and acoustical parts, the second-order problem which is considered is $[9,35]$

$$
-\omega^{2}[M]\{q\}+i \omega[C]\{q\}+[K]\{q\}=\{f\},
$$

where $[C]=\left[\begin{array}{cc}C_{S} & 0 \\ 0 & C_{F}\end{array}\right], C_{S}$ and $C_{F}$ being non-negative, definite, symmetric matrices. This implies in particular that no loss occur over the coupling area. This is valid while no absorbing material is glued on the coupling surface. Cases with porous layers attached to the vibrating structure have not yet been investigated by the authors. In the following, eq. (2) will be considered as the reference problem.

The direct quadratic eigenvalue problem associated to this equation is

$$
\left([M] \lambda_{j}^{2}+[C] \lambda_{j}+[K]\right)\left\{\phi_{R j}\right\}=0
$$

and the corresponding adjoint eigenvalue problem writes

$$
\left([M]^{T} \lambda_{j}^{2}+[C]^{T} \lambda_{j}+[K]^{T}\right)\left\{\phi_{L j}\right\}=0,
$$

where $\lambda_{j}$ is the $j$-th eigenvalue associated to the $j$-th right eigenvector $\left\{\phi_{R j}\right\}$ and $j$-th left eigenvector $\left\{\phi_{L j}\right\}$.

Since the matrices are not symmetric, the eigenvalues of the problem are real or come in pairs $\left(\lambda_{j}, \lambda_{j}^{*}\right)$. If $\left\{\phi_{j}\right\}$ is a (right or left) eigenvector associated to $\lambda_{j}$, then $\left\{\phi_{j}^{*}\right\}$ is a (right or left) eigenvector associated to $\lambda_{j}^{*}$. A very complete review of this kind of problem is addressed in [36].

\subsection{Complex modes and modal decomposition of the permanent harmonic response}

The eigenmodes of the system can be efficiently used in particular for the modal decomposition of the permanent harmonic response. This can be done considering the state-space representation of the system:

$$
\left[\begin{array}{cc}
C & M \\
M & 0
\end{array}\right]\left\{\begin{array}{c}
\dot{q}(t) \\
\ddot{q}(t)
\end{array}\right\}-\left[\begin{array}{cc}
-K & 0 \\
0 & M
\end{array}\right]\left\{\begin{array}{c}
q(t) \\
\dot{q}(t)
\end{array}\right\}=\left\{\begin{array}{c}
f(t) \\
0
\end{array}\right\} .
$$

The eigenvalues of this problem can be stored in the spectral matrix

$$
[\Lambda]=\left[\lambda_{j}\right] .
$$

The $j$-th eigenvalue is associated to: 
- a right eigenvector $\left\{\begin{array}{c}\phi_{R j} \\ \phi_{R j} \lambda_{j}\end{array}\right\}$. Storing the eigenvectors (in the same order as the eigenvalues) in the modal matrix $\left[\begin{array}{c}\phi_{R} \\ \phi_{R} \Lambda\end{array}\right]$, the following relationship is verified:

$$
\left[\begin{array}{cc}
C & M \\
M & 0
\end{array}\right]\left[\begin{array}{c}
\phi_{R} \\
\phi_{R} \Lambda
\end{array}\right]\left[\lambda_{j}\right]=\left[\begin{array}{cc}
-K & 0 \\
0 & M
\end{array}\right]\left[\begin{array}{c}
\phi_{R} \\
\phi_{R} \Lambda
\end{array}\right]
$$

- a left eigenvector $\left\{\begin{array}{c}\phi_{L j} \\ \phi_{L j} \lambda_{j}\end{array}\right\}$. Storing the eigenvectors (in the same order as the eigenvalues) in the modal matrix $\left[\begin{array}{c}\phi_{L} \\ \phi_{L} \Lambda\end{array}\right]$, the following relationships are verified:

$$
\left[\begin{array}{cc}
C & M \\
M & 0
\end{array}\right]^{T}\left[\begin{array}{c}
\phi_{L} \\
\phi_{L} \Lambda
\end{array}\right]\left[\lambda_{j}\right]=\left[\begin{array}{cc}
-K & 0 \\
0 & M
\end{array}\right]^{T}\left[\begin{array}{c}
\phi_{L} \\
\phi_{L} \Lambda
\end{array}\right]
$$

or

$$
\left[\lambda_{j} \backslash\left[\begin{array}{c}
\phi_{L} \\
\phi_{L} \Lambda
\end{array}\right]^{T}\left[\begin{array}{cc}
C & M \\
M & 0
\end{array}\right]=\left[\begin{array}{c}
\phi_{L} \\
\phi_{L} \Lambda
\end{array}\right]^{T}\left[\begin{array}{cc}
-K & 0 \\
0 & M
\end{array}\right]\right.
$$

The orthogonality relationships can be written using $2 n$ arbitrary values to build the diagonal matrix $[\xi]=\left[\xi_{j}\right]$ :

$$
\left[\begin{array}{c}
\phi_{L} \\
\phi_{L} \Lambda
\end{array}\right]^{T}\left[\begin{array}{cc}
C & M \\
M & 0
\end{array}\right]\left[\begin{array}{c}
\phi_{R} \\
\phi_{R} \Lambda
\end{array}\right]=\left[\zeta_{\xi_{\curlywedge}}\right] \text { or }\left[\begin{array}{c}
\phi_{L} \\
\phi_{L} \Lambda
\end{array}\right]^{T}\left[\begin{array}{cc}
-K & 0 \\
0 & M
\end{array}\right]\left[\begin{array}{c}
\phi_{R} \\
\phi_{R} \Lambda
\end{array}\right]=\left[\xi_{\zeta_{j}} \lambda_{j}\right]
$$

The modal decomposition of the permanent harmonic response at frequency $\omega$ is finally:

$$
\left\{\begin{array}{c}
q(t) \\
\dot{q}(t)
\end{array}\right\}=\left[\begin{array}{c}
\phi_{R} \\
\phi_{R} \Lambda
\end{array}\right]\left[\backslash \frac{1}{\xi_{j}\left(i \omega-\lambda_{j}\right)}\right]\left[\begin{array}{c}
\phi_{L} \\
\phi_{L} \Lambda
\end{array}\right]^{T}\left\{\begin{array}{c}
f(\omega) \\
0
\end{array}\right\} e^{i \omega t},
$$

where $f(\omega)$ is the complex amplitude of the harmonic excitation. This relationship can also be written using the $n$ degrees of freedom notations in the frequency domain:

$$
\{q(\omega)\}=\left[\phi_{R}\right]\left[\backslash \frac{1}{\xi_{j}\left(i \omega-\lambda_{j}\right)}\right]\left[\phi_{L}\right]^{T}\{f(\omega)\},
$$

In the field of experimental modal analysis, an experimental reduced model is built from complex modes, which are identified using FRFs (Frequency Response Functions) with techniques adapted from those classically used for symmetric problems [31].

Here, for practical reasons and without loss of generality, one will assume that the eigenshapes are normalized such as $\xi_{j}=1, \forall j=1 \ldots 2 n$.

\subsection{Particular case of vibroacoustics}

In the case of vibroacoustic modes, due to the special topology of the problem, there is a direct link between the left and right complex modes [9, 35]. This relationship is related to the type of damping which is considered in the problem. In the considered case, with only viscous 
damping and without structural/fluid cross damping, if $\left\{\phi_{R j}\right\}=\left\{\begin{array}{c}v_{j} \\ \varphi_{j}\end{array}\right\}$ is a right eigenvector corresponding to $\lambda_{j}$ eigenvalue ( $v$ corresponds to the structural dofs, $\varphi$ to the acoustic dofs), then it can easily be demonstrated that $\left\{\phi_{L j}\right\}=\left\{\begin{array}{c}v_{j} \\ -\varphi_{j} / \lambda_{j}^{2}\end{array}\right\}$. If the damping has any other expression, the relationship is no longer valid. To the author's knowledge, this has not been indicated before in the open literature related to vibroacoustical modal analysis. It can be demonstrated by considering a full damping matrix $[C]$ : in this case the right modal equation indicates that

$$
\left(\left[\begin{array}{cc}
M_{S} & 0 \\
L^{T} & M_{F}
\end{array}\right] \lambda_{j}^{2}+\left[\begin{array}{cc}
C_{S} & C_{S F} \\
C_{F S} & C_{F}
\end{array}\right] \lambda_{j}+\left[\begin{array}{cc}
K_{S} & -L \\
0 & K_{F}
\end{array}\right]\right)\left\{\begin{array}{c}
v_{j} \\
\varphi_{j}
\end{array}\right\}=0 .
$$

Using $\left\{\phi_{L j}\right\}=\left\{\begin{array}{c}v_{j} \\ -\varphi_{j} / \lambda_{j}^{2}\end{array}\right\}$ in the adjoint equation yields

$$
\begin{aligned}
\left([M]^{T}\right. & \left.\lambda_{j}^{2}+[C]^{T} \lambda_{j}+[K]^{T}\right)\left\{\phi_{L j}\right\} \\
& =\left(\left[\begin{array}{cc}
M_{S} & L \\
0 & M_{F}
\end{array}\right] \lambda_{j}^{2}+\left[\begin{array}{cc}
C_{S} & C_{F S}^{T} \\
C_{S F}^{T} & C_{F}
\end{array}\right] \lambda_{j}+\left[\begin{array}{cc}
K_{S} & 0 \\
-L^{T} & K_{F}
\end{array}\right]\right)\left\{\begin{array}{c}
v_{j} \\
-\varphi_{j} / \lambda_{j}^{2}
\end{array}\right\} \\
& =\left\{\begin{array}{c}
-\left(\frac{1}{\lambda_{j}} C_{F S}^{T}+\lambda_{j} C_{S F}\right) \varphi_{j} \\
\left(\frac{1}{\lambda_{j}} C_{F S}+\lambda_{j} C_{S F}^{T}\right) v_{j}
\end{array}\right\},
\end{aligned}
$$

which can be zero for all $j$ only if $C_{F S}=0$ and $C_{F S}=0$. For more general damping considerations, in particular if the structural part or the fluid domain exhibits another type of damping (hysteretic or any other frequency-dependent expression), the special relationship between left and right eigenvectors holds until there is no cross term in the damping matrix. Nevertheless, in this case the modes can no longer be evaluated from a quadratic eigenvalue problem.

The relationship can finally be expressed as

$$
\text { If }\left[\phi_{R}\right]=\left[\begin{array}{c}
v \\
\varphi
\end{array}\right] \text { then }\left[\phi_{L}\right]=\left[\begin{array}{c}
v \\
-\varphi \Lambda^{-2}
\end{array}\right] \text {, }
$$

and the modal normalization factor $\xi_{j}$ can be written

$$
\xi_{j}=\frac{1}{\lambda_{j}}\left(v_{j}^{T}\left(M_{S} \lambda_{j}^{2}-K_{S}\right) v_{j}-\frac{1}{\lambda_{j}^{2}} \varphi_{j}^{T}\left(M_{F} \lambda_{j}^{2}-K_{F}\right) \varphi_{j}\right) .
$$

\section{Correlation criterion}

In verification and validation procedures of modal models, correlation criterion are required to check the similarity of modes and for modes pairing. This avoids association of non-physically corresponding modes between two modal bases (model/test, model/model or test/test). When dealing with experimental models, auto-correlation criterion can be used to check the ability of the sensor placement to distinguish the modes of interest. In this section, a suitable criteria is proposed for vibroacoustic applications. The modes of interest are the complex ones defined in section 2.2. 
In structural dynamics, a very common tool for correlation of two modal bases is the MAC criterion and the associated weighted MAC criterion [37]:

$$
W M A C(\phi, \psi)=\frac{\left(\phi^{H} W \psi\right)^{2}}{\left(\phi^{H} W \phi\right)\left(\psi^{H} W \psi\right)},
$$

where $\phi$ and $\psi$ are the two vectors of interest, $W$ is a weighting matrix and ${ }^{H}$ is used for the hermitian (conjugate transpose) of a vector. Using the identity matrix for $W$ gives the MAC criterion. The correlation between two sets of vectors can then be done using the criterion in a matrix representation. In structural dynamics without damping, if the two bases are identical, the weighted MAC matrix is theoretically equal to unity if the mass (or stiffness) matrix is used for $W$. Using the classical (unweighted) MAC, the matrix can be close to unity if the physical mass is well distributed in space. These indicators can not be directly applied for non self adjoint problems, but the criteria can easily be extended in presence of left and right eigenvectors:

$$
W \operatorname{LRMAC}\left(\phi_{L, R}, \psi_{L, R}\right)=\frac{\left(\phi_{L}^{H} W \psi_{R}\right)\left(\psi_{L}^{H} W \phi_{R}\right)}{\left(\phi_{L}^{H} W \phi_{R}\right)\left(\psi_{L}^{H} W \psi_{R}\right)} .
$$

At this point, it should be mentionned that instead of this physical-state definition of the MAC, the orthogonality relationships (10) may be used to define a MAC in the state-space. This would lead to a mathematically exact correlation index, even in the case of high damping. However, it has been observed in the practical applications that the indicator (18) gives good results, as it will be shown later in the paper. Moreover this is coherent with the classical approach in structural dynamics where only the physical-state version (17) is commonly used.

Suitable correlation criteria for multiphysics systems are very little discussed in literature [38]. The use of a coherent weighting matrix is of first importance for multiphysics applications, in particular when the orders of magnitude of the chosen physical variables are different, which is typically the case in most of practical problems in vibroacoustics. Even for pure structural dynamics applications, when rotation dofs are mixed with translation dofs, some troubles can appear. However, in practice, the MAC value is often evaluated using only translation dofs which explains the fact that the unweighted version of the MAC is generally used.

For the vibroacoustic case considered in this paper, the use of a weighting matrix is mandatory. The direct use of classical MAC value, on all dofs, or even on only translation or pressure dofs, could lead to errors in correlation. The figure 2 shows the auto-MAC (auto-correlation) of an exact vibroacoustic basis for these 3 cases. This example is taken from a parallelipedic acoustic cavity closed by a flexible plate, but the authors have found equivalent conclusions on various systems: it is clear that the correlation information is not appropriate, and that erroneous results could be obtained using one of these criteria. It can also be observed that the MAC value estimated using all dofs is almost the same as the one obtained by considering only pressures dofs, since the order of magnitude of pressure is much larger than the one of the displacement.

Using the WLRMAC value with $W=M$ leads to the identity matrix, as shown in figure $3 \mathrm{a}$. In practical applications involving experimental results, only a reduced set of dofs are equipped with sensors, and a model reduction technique (typically, a Guyan reduction) can be used to estimate the mass matrix reduced to the points of interest. In fully experimental situations, when no model is available, a simplified lumped matrix $\tilde{M}$ can be defined to avoid the masking of structural dofs by acoustic dofs. $\tilde{M}$ can be chosen as a diagonal matrix with unit terms for translation dofs and a constant factor $f$ for acoustic dofs. As shown in figure $3 \mathrm{~b}$, the choice of $f=\frac{\left\langle U^{2}\right\rangle}{\left\langle P^{2}\right\rangle}$, where 

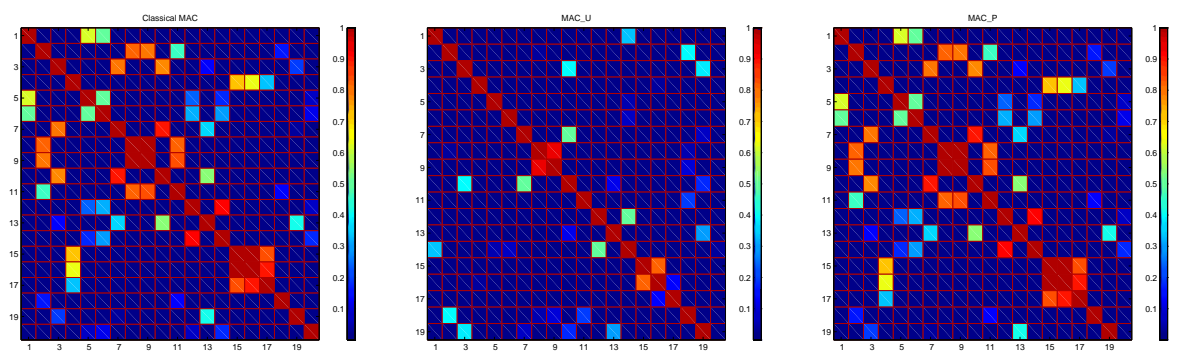

Figure 2: a) Classical auto-MAC (using all dofs), b) auto-MAC using only $U$ dofs, c) auto-MAC using only $P$ dofs

$<\cdot>$ indicates the mean value, leads to an auto-correlation close to identity if the mass is well distributed on the structure, which is the behavior of the classical MAC criterion.
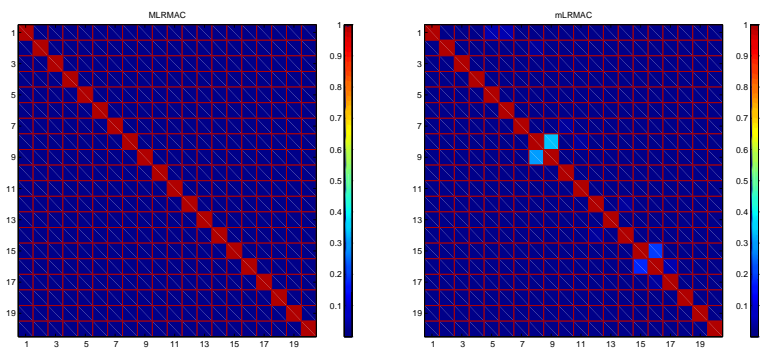

Figure 3: a) auto-MLRMAC, b) auto-MLRMAC with lumped mass matrix

\section{Identification of experimental reduced models}

\subsection{General case}

Following the approach described in [31], it is possible to determine an experimental reduced model from the knowledge of $N$ modes measured on $N$ degrees of freedom. The general procedure is briefly recalled here.

For a general nonsymmetric second-order problem, the orthogonality relations can be inverted in order to obtain the matrices of the system:

$$
\begin{aligned}
{\left[\begin{array}{cc}
C & M \\
M & 0
\end{array}\right]^{-1} } & =\left[\begin{array}{cc}
0 & M^{-1} \\
M^{-1} & -M^{-1} C M^{-1}
\end{array}\right] \\
& =\left[\begin{array}{cc}
\phi_{R} \phi_{L}^{T} & \phi_{R} \Lambda \phi_{L}^{T} \\
\phi_{R} \Lambda \phi_{L}^{T} & \phi_{R} \Lambda^{2} \phi_{L}^{T}
\end{array}\right],
\end{aligned}
$$


and

$$
\begin{aligned}
{\left[\begin{array}{cc}
-K & 0 \\
0 & M
\end{array}\right]^{-1} } & =\left[\begin{array}{cc}
-K^{-1} & 0 \\
0 & M^{-1}
\end{array}\right] \\
& =\left[\begin{array}{cc}
\phi_{R} \Lambda^{-1} \phi_{L}^{T} & \phi_{R} \phi_{L}^{T} \\
\phi_{R} \phi_{L}^{T} & \phi_{R} \Lambda \phi_{L}^{T}
\end{array}\right],
\end{aligned}
$$

yielding

$$
\begin{gathered}
M=\left[\phi_{R} \Lambda \phi_{L}^{T}\right]^{-1}, \\
K=-\left[\phi_{R} \Lambda^{-1} \phi_{L}^{T}\right]^{-1}, \\
C=-\left[M \phi_{R} \Lambda^{2} \phi_{L}^{T} M\right] .
\end{gathered}
$$

These relationships are valid only if the so-called properness condition is verified:

$$
\left[\phi_{R} \phi_{L}^{T}\right]=0 \text {. }
$$

The complex modes of the system must then verify this relation if they constitute the full basis of a physical system.

\subsection{Particular topology of matrices for vibroacoustic coupling}

Since the considered vibroacoustic problem exhibits specific matrices topology, additional relationships must be verified by the complex modes. Indeed, Eq. (24) can be expressed using only the right eigenvectors since left ones can be deduced from them:

$$
\left[\begin{array}{ll}
v v^{T} & -v \Lambda^{-2} \varphi^{T} \\
\varphi v^{T} & -\varphi \Lambda^{-2} \varphi^{T}
\end{array}\right]=0
$$

The equation (25) is not sufficient to insure that the topology of the reconstructed matrices is the same as for the initial vibroacoustic system (1). In particular, the structural and acoustic blocks can be found using the partition of the eigenvectors:

$$
M^{-1}=\left[\begin{array}{cc}
M_{S}^{-1} & 0 \\
-M_{F}^{-1} L^{T} M_{S}^{-1} & M_{F}^{-1}
\end{array}\right]=\phi_{R} \Lambda \phi_{L}^{T}=\left[\begin{array}{cc}
v \Lambda v^{T} & -v \Lambda^{-1} \varphi^{T} \\
\varphi \Lambda v^{T} & -\varphi \Lambda^{-1} \varphi^{T}
\end{array}\right] .
$$

In the same way, one has

$$
-K^{-1}=\left[\begin{array}{cc}
-K_{S}^{-1} & -K_{S}^{-1} L K_{F}^{-1} \\
0 & -K_{F}^{-1}
\end{array}\right]=\phi_{R} \Lambda^{-1} \phi_{L}^{T}=\left[\begin{array}{cc}
v \Lambda^{-1} v^{T} & -v \Lambda^{-3} \varphi^{T} \\
\varphi \Lambda^{-1} v^{T} & -\varphi \Lambda^{-3} \varphi^{T}
\end{array}\right],
$$

and

$$
\left[\begin{array}{cc}
-M_{S}^{-1} C_{S} M_{S}^{-1} & 0 \\
M_{F}^{-1} L^{T} M_{S}^{-1} C_{S} M_{S}^{-1}+M_{F}^{-1} C_{F} M_{F}^{-1} L^{T} M_{S}^{-1} & -M_{F}^{-1} C_{F} M_{F}^{-1}
\end{array}\right]=\left[\begin{array}{cc}
v \Lambda^{2} v^{T} & -v \varphi^{T} \\
\varphi \Lambda^{2} v^{T} & -\varphi \varphi^{T}
\end{array}\right] .
$$

One can resume the set of equations using a distinction between the expression of identified matrices

$$
\left\{\begin{array}{l}
M_{S}=\left(v \Lambda v^{T}\right)^{-1} \\
M_{F}=-\left(\varphi \Lambda^{-1} \varphi^{T}\right)^{-1} \\
L=-M_{S} v \Lambda \varphi^{T} M_{F}=\left(v \Lambda v^{T}\right)^{-1} v \Lambda \varphi^{T}\left(\varphi \Lambda^{-1} \varphi^{T}\right)^{-1} \\
K_{S}=-\left(v \Lambda^{-1} v^{T}\right)^{-1} \\
K_{F}=\left(\varphi \Lambda^{-3} \varphi^{T}\right)^{-1} \\
C_{S}=-M_{S} v \Lambda^{2} v^{T} M_{S}=-\left(v \Lambda v^{T}\right)^{-1} v \Lambda^{2} v^{T}\left(v \Lambda v^{T}\right)^{-1} \\
C_{F}=M_{F} \varphi \varphi^{T} M_{F}=\left(\varphi \Lambda^{-1} \varphi^{T}\right)^{-1} \varphi \varphi^{T}\left(\varphi \Lambda^{-1} \varphi^{T}\right)^{-1} \\
10
\end{array}\right.
$$


and the relationships that the complex eigenmodes must verify:

$$
\left\{\begin{array}{l}
v v^{T}=0 \\
v \varphi^{T}=0 \\
v \Lambda^{-2} \varphi^{T}=0 \\
\varphi \Lambda^{-2} \varphi^{T}=0 \\
v \Lambda^{-1} \varphi^{T}=0 \\
\left(v \Lambda v^{T}\right)^{-1} v \Lambda \varphi^{T}\left(\varphi \Lambda^{-1} \varphi^{T}\right)^{-1}+\left(v \Lambda^{-1} v^{T}\right)^{-1} v \Lambda^{-3} \varphi^{T}\left(\varphi \Lambda^{-3} \varphi^{T}\right)^{-1}=0 \\
\varphi \Lambda v^{T}\left(v \Lambda v^{T}\right)^{-1} v \Lambda^{2} v^{T}+\varphi \varphi^{T}\left(\varphi \Lambda^{-1} \varphi^{T}\right)^{-1} \varphi \Lambda v^{T}-\varphi \Lambda^{2} v^{T}=0
\end{array}\right.
$$

The four first equations of system (30) correspond to the properness condition in the state-space (25); the fifth one to the topology of the mass and stifness matrices (block extradiagonal null terms); the sixth one indicates that coupling terms in mass and stiffness matrices are transposed one from another; and the last one corresponds to the null block extradiagonal terms in the damping matrix.

\subsection{Optimal correction of complex modes}

When the complex modes are available from experimental identification, one can use the previous relationships to find the reduced model which is supposed to have the same behavior as the measured one. The fact is that in general, the modes do not verify the constraints equations (30), which results in a bad conditioning of the inverse procedure. It has been shown [30, 31] that imposing the properness condition on complex modes performs an indirect regularization of the problem. The approach consists in correcting the identified complex eigenvectors in order that they verify the required constraints, before applying the inverse procedure. In practice, this generally leads to small phase correction on the vectors and almost no change in amplitudes, which is coherent with experimental considerations [31].

Constraints equations (30) have more complex expressions than in the general case considered in [31]. This is due to the special topology of the problem. It is then impossible to find an explicit solution of the optimization problem using a Ricatti equation.

The regularization requires the resolution of a constrained optimization problem which can be written as follows. Starting from the knowledge of experimental eigenmodes (eigenvectors with structural dofs $v^{e}$, acoustic dofs $\varphi^{e}$ and eigenvalues $\Lambda$ ):

$$
\begin{aligned}
& \text { Find } v \text { and } \varphi \text { minimizing } f_{1}=\left\|v-v^{e}\right\| \text { and } f_{2}=\left\|\varphi-\varphi^{e}\right\| \\
& \text { while }\left\{\begin{array}{l}
g_{1}=v v^{T}=0 \\
g_{2}=v \varphi^{T}=0 \\
g_{3}=v \Lambda^{-2} \varphi^{T}=0 \\
g_{4}=\varphi \Lambda^{-2} \varphi^{T}=0 \\
g_{5}=v \Lambda^{-1} \varphi^{T}=0 \\
g_{6}=\left(v \Lambda v^{T}\right)^{-1} v \Lambda \varphi^{T}\left(\varphi \Lambda^{-1} \varphi^{T}\right)^{-1}+\left(v \Lambda^{-1} v^{T}\right)^{-1} v \Lambda^{-3} \varphi^{T}\left(\varphi \Lambda^{-3} \varphi^{T}\right)^{-1}=0 \\
g_{7}=\varphi \Lambda v^{T}\left(v \Lambda v^{T}\right)^{-1} v \Lambda^{2} v^{T}+\varphi \varphi^{T}\left(\varphi \Lambda^{-1} \varphi^{T}\right)^{-1} \varphi \Lambda v^{T}-\varphi \Lambda^{2} v^{T}=0 .
\end{array}\right.
\end{aligned}
$$

Solving this problem is clearly not obvious, because the constraints are not verified by the experimental vectors. Moreover the constraints are matrix constraints. One of the possibilities consists in reformulating it as:

Find $v$ and $\varphi$ minimizing $f_{1}=\left\|v-v^{e}\right\|$ and $f_{2}=\left\|\varphi-\varphi^{e}\right\|$ while $\left\|g_{\alpha}\right\|-\tau_{\alpha} \leq 0, \forall \alpha=1 \ldots 7$ 
where $\tau_{\alpha}$ are arbitrary (small) thresholds and $\|\cdot\|$ is the Frobenius norm.

Instead of using time-consuming optimization algorithms, we have investigated several ways allowing to approximate the solutions of this problem.

\subsubsection{General nonsymmetric case}

A simplified approach consists in not considering the particular topology of the matrices. This limits the constraints and leads to this formulation of the optimization procedure:

$$
\begin{aligned}
& \text { Find } v \text { and } \varphi \text { minimizing } f_{1}=\left\|v-v^{e}\right\| \text { and } f_{2}=\left\|\varphi-\varphi^{e}\right\| \\
& \text { while }\left\{\begin{array}{l}
g_{1}=v v^{T}=0 \\
g_{2}=v \varphi^{T}=0 \\
g_{3}=v \Lambda^{-2} \varphi^{T}=0 \\
g_{4}=\varphi \Lambda^{-2} \varphi^{T}=0 .
\end{array}\right.
\end{aligned}
$$

It can be reformulated using a Hamilton function $H$, with 4 matrices of real Lagrange multipliers $\delta_{i}(i=1$ to 4$)$, written as

$$
\begin{aligned}
H= & \left\|v-v^{e}\right\|+\alpha\left\|\varphi-\varphi^{e}\right\| \\
& +\delta_{1} \otimes v v^{T}+\delta_{2} \otimes v \varphi^{T} \\
& +\delta_{3} \otimes v \Lambda^{-2} \varphi^{T}+\delta_{4} \otimes \varphi \Lambda^{-2} \varphi^{T} .
\end{aligned}
$$

Minimization of $H$ yields

$$
\begin{array}{ll}
0=\left\{\begin{array}{l}
v \\
\varphi
\end{array}\right\}-\left\{\begin{array}{l}
v^{e} \\
\varphi^{e}
\end{array}\right\} & +\frac{1}{2}\left[\begin{array}{cc}
\delta_{1}+\delta_{1}^{T} & \delta_{2} \\
\delta_{2}^{T} & 0
\end{array}\right]\left\{\begin{array}{l}
\bar{v} \\
\bar{\varphi}\}
\end{array}\right. \\
& +\frac{1}{2}\left[\begin{array}{cc}
0 & \delta_{3} \\
\delta_{3}^{T} & \delta_{4}+\delta_{4}^{T}
\end{array}\right]\left\{\frac{v \Lambda^{-2}}{\varphi \Lambda^{-2}}\right\} \\
v v^{T}=0 & \begin{array}{l}
v \varphi^{T}=0 \\
v \Lambda^{-2} \varphi^{T}=0 \\
\varphi \Lambda^{-2} \varphi^{T}=0 .
\end{array}
\end{array}
$$

Note that this system is coherent with the ones proposed in [30, 31]. Nevertheless since it involves matrices in a nonlinear way, the numerical procedures required to solve it are still too expensive to be compatible with real-time applications during modal analysis procedure. This motivated further investigations for approximate resolutions.

\subsubsection{Diagonal enforcement of properness condition}

A simple way to enforce partially the properness condition consists in considering only the diagonal terms of the properness (25). This leads to the two following decoupled problems:

$$
\begin{aligned}
& \text { Find } v \text { minimizing } f_{1}=\left\|v-v^{e}\right\| \\
& \text { while } g_{1}=v v^{T}=0 ; \\
& \text { Find } \varphi \text { : minimizing } f_{2}=\left\|\varphi-\varphi^{e}\right\| \\
& \text { while } g_{4}=\varphi \Lambda^{-2} \varphi^{T}=0 \text {. }
\end{aligned}
$$

Resolution of (36) is easy using the procedure described in [30]. (37) can also be solved by using this procedure with unknown vector $\tilde{\varphi}=i \varphi \Lambda^{-1}$. The constraint is then $\tilde{\varphi} \tilde{\varphi}^{T}=0$, which is the expression required by the procedure. 
This first simple approach will be compared with others in the following examples. The next part introduces an alternative way for partial enforcement of the properness condition.

\subsubsection{Over-enforcement of the properness condition}

The procedure proposed in [30] being valid for any problem with a constraint of the form $x x^{T}=0$, it is possible to use

$$
x=\left[\begin{array}{c}
v \\
\varphi \\
-\varphi \Lambda^{2}
\end{array}\right]
$$

so

$$
x x^{T}=\left[\begin{array}{ccc}
v v^{T} & v \varphi^{T} & -v \Lambda^{2} \varphi^{T} \\
\varphi v^{T} & \varphi \varphi^{T} & -\varphi \Lambda^{2} \varphi^{T} \\
-\varphi \Lambda^{2} v^{T} & -\varphi \Lambda^{2} \varphi^{T} & \varphi \Lambda^{4} \varphi^{T}
\end{array}\right] .
$$

The four terms of (35) are included in this matrix, but correcting the eigenvectors with this constraint will result in imposing two non required conditions, namely $\varphi \varphi^{T}=0$ and $\varphi \Lambda^{4} \varphi^{T}=0$. Moreover, this will imply that $\varphi$ and $\varphi \Lambda^{2}$ are independent, which means that eigenvalues will possibly be also modified. Nevertheless, we will see in the next section that this procedure gives interesting results.

\subsubsection{Numerical illustration}

In order to illustrate the impact and efficiency of the optimal correction, the following procedure is applied:

1. Starting from given Frequency Response Functions (FRFs), complex modes are identified through least square complex curve fitting. Corresponding complex eigenvalues and eigenvectors are used as starting point for the correction.

2. These complex modes are used in the inverse procedure to identify system matrices. These matrices are then used to compute the FRFs of the system using eq. (29), which can be compared to the initial ones. This first identification process is called "direct". Differences between FRFs are due to the fact that the identified complex modes do not verify the relations (30).

3. The "diagonal" correction procedure is performed on the eigenvectors before the application of the inverse procedure. Matrices are identified and FRFs are computed again. This technique is called "diagonal" properness enforcement.

4. Equivalent work is done after application of the "over" enforcement of the properness on the complex modes.

A very simple numerical test-case is first considered to illustrate the principle of the approach The system has 2 degrees of freedom and a topology coherent with the vibroacoustic formulation:

$$
\begin{aligned}
& {\left[\begin{array}{cc}
3.234 & 0 \\
-1.465 & 1.275 \times 10^{-2}
\end{array}\right]\left\{\begin{array}{l}
\ddot{x} \\
\ddot{p}
\end{array}\right\}+\left[\begin{array}{cc}
1.123 & 0 \\
0 & 3.185 \times 10^{-3}
\end{array}\right]\left\{\begin{array}{l}
\dot{x} \\
\dot{p}
\end{array}\right\} } \\
& 13+\left[\begin{array}{cc}
1000 & 1.465 \\
0 & 1.65
\end{array}\right]\left\{\begin{array}{l}
x \\
p
\end{array}\right\}=0 .
\end{aligned}
$$


The numerical values of the terms in the matrices have been chosen randomly, with the constraint that the order of magnitude of the various terms are in accordance with a physical system exhibiting two modes in the low frequency range. Many other "random" cases have been investigated, leading to similar conclusions. The eigenvalues of the system described by (40) are $\lambda_{1}=-0.1065+10.16 i$ and $\lambda_{2}=-0.1920+19.68 i$, hence

$$
\phi_{R}=\left[\begin{array}{cc}
-0.0266+0.0263 i & 0.0597-0.0598 i \\
12.1474-11.89 i & 10.39-10.22 i
\end{array}\right] .
$$

In order to improve readability, the conjugate terms have been omitted in this expression. In the calculations, the matrix $\left[\phi_{R}\right]$ has 2 lines and 4 columns.

$10 \%$ uniform random noise has then been added on the real and imaginary parts of each component of the eigenvectors. One realization of this statistical process is (we use the $D$ index to refer to the direct identification)

$$
\phi_{R}^{D}=\left[\begin{array}{cc}
-0.0274+0.0256 i & 0.0599-0.0614 i \\
12.5937-11.6587 i & 10.0916-10.0166 i
\end{array}\right] .
$$

This high noise level allows testing of the robustness of the procedure. The properness matrix is then:

$$
\phi_{R}^{D} \phi_{L}^{D T}=\left[\begin{array}{cc}
-0.0002 & -0.0008 \\
-0.1139 & 0.3066
\end{array}\right] .
$$

Using the "diagonal" procedure leads to

$$
\phi_{R}^{G}=\left[\begin{array}{cc}
-0.0275+0.0255 i & 0.0602-0.0611 i \\
12.3188-11.9366 i & 9.8712-10.2546 i
\end{array}\right],
$$

which corresponds to the properness matrix:

$$
\phi_{R}^{G} \phi_{L}^{G T}=\left[\begin{array}{cc}
0 & -6.99 \times 10^{-4} \\
-0.1345 & -8.9 \times 10^{-16}
\end{array}\right] .
$$

Compared to (43), it is clear that the procedure works as awaited.

Next, "over" enforcement is performed on the vectors, resulting in

$$
\phi_{R}^{O}=\left[\begin{array}{cc}
-0.0254+0.0275 i & 0.0611-0.0602 i \\
12.2942-11.9498 i & 9.8516-10.2667 i
\end{array}\right],
$$

which corresponds to

$$
\phi_{R}^{O} \phi_{L}^{O T}=\left[\begin{array}{cc}
-3.3 \times 10^{-16} & -8.4 \times 10^{-17} \\
-2.0 \times 10^{-15} & 1.3 \times 10^{-15}
\end{array}\right]
$$

The $O$ index is used to refer to this case. The full matrix is now close to zero, which is coherent.

Matrices identification is now possible using equations (21) to (23). Mass matrices are

$$
\begin{gathered}
M_{D}=\left[\begin{array}{cc}
3.113 & 0.0001 \\
-1.403 & 0.0127
\end{array}\right], M_{G}=\left[\begin{array}{cc}
3.113 & 0.0001 \\
-1.402 & 0.0127
\end{array}\right], \\
M_{O}=\left[\begin{array}{cc}
3.118 & 0.0001 \\
-1.404 & 0.0127 \\
14 &
\end{array}\right.
\end{gathered}
$$


Stiffness matrices are

$$
\begin{gathered}
K_{D}=\left[\begin{array}{ll}
986.1 & 1.465 \\
5.047 & 1.641
\end{array}\right], K_{G}=\left[\begin{array}{ll}
986.1 & 1.466 \\
5.118 & 1.641
\end{array}\right], \\
K_{O}=\left[\begin{array}{ll}
987.4 & 1.465 \\
4.991 & 1.641
\end{array}\right] .
\end{gathered}
$$

Finally, the damping matrices are

$$
\begin{gathered}
C_{D}=\left[\begin{array}{cc}
0.4310 & -0.0044 \\
-1.151 & 0.0099
\end{array}\right], \quad C_{G}=\left[\begin{array}{cc}
1.108 & -0.0053 \\
-1.227 & 0.0029
\end{array}\right], \\
C_{O}=\left[\begin{array}{cc}
2.228 & -0.0042 \\
-0.5836 & 0.0004
\end{array}\right] .
\end{gathered}
$$

It is then clear that the three procedures give almost the same values of mass and stiffness matrices. The topology is not exactly the same as the initial system: the terms that should be null are small, and the extradiagonal stiffness and mass terms that should be equal exhibit less than 5\% of difference. Much larger differences are observed in the damping matrix: the direct procedure gives the most erroneous values, while the corrected vectors result in more pertinent values. The "diagonal" procedure gives correct values of the diagonal terms, but "large" extradiagonal terms are estimated, while the "over" procedure gives almost twice damping on the diagonal and extradiagonal terms are reduced. It should be emphasized that the starting data are the noisy vectors, which do not necessarily correspond to any second-order physical system. Moreover, the addition of noise on the vectors could result in changes in the apparent stiffness, mass or damping of the system, which possibly explains the differences observed on the values in the damping matrices. The objective of the methodology is to determine the physical system which exhibits a behavior as close as possible to the one corresponding to the identified modal basis (the "noisy" one in our case). The damping matrix identification procedure is then highly sensitive to noise, and the correction procedures act as indirect regularizations.

Once these matrices are identified, the FRFs of each system can be computed. Figure 4 gives the collocated structural FRFs computed from:

- the modal synthesis using the original vectors with added noise, this is the reference,

- the identified matrices (without properness enforcement, and with the two procedures described before).

This figure illustrates the fact that the direct identification does not allow correct estimation of the dynamics of the system, in particular on the phase of the signals, while the two results obtained after optimal correction lead to better estimation of the responses. The only procedure that reproduces correctly the damping effect at resonances is the "over" technique.

In this 2-dofs case, the numerical cost of the procedure is obviously very low. For larger problems, the cost is mainly associated to the resolution of the Riccati equation, which remains low compared to global constrained optimization techniques that would be required for solving the problem (31). In reference [31], some details are given about the practical implementation 


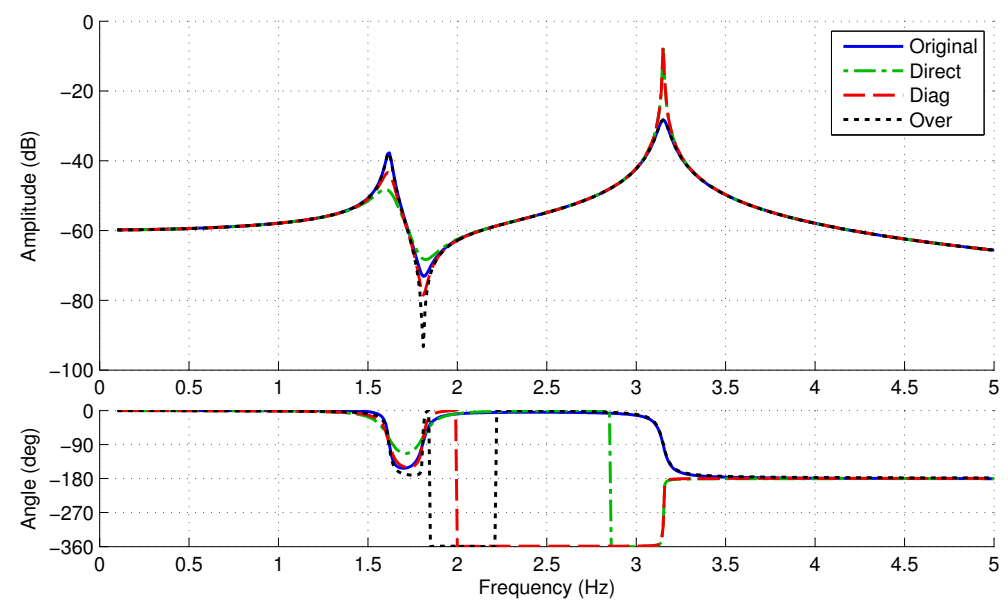

Figure 4: Comparison of FRFs estimated from corrected eigenvectors on simulated data

of the Riccati solver and associated numerical issues. This is very coherent with the fact that for practical applications, it is not expected that the number of degrees of freedom is very large, because of measurement limits: each degree of freedom has to be measured. This limitation could be overcome in the next years thanks to the deployment of various measurement devices with high spatial resolution.

\section{Experimental validation of the procedure}

\subsection{Description of the system}

In this part, an experimental illustration of the procedure is proposed. Experiments are conducted on the so-called "small cabin" of LVA-INSA Lyon, composed by a parallelepipedic cavity closed by a flexible plate, as shown on figure 5. Several plates being available, the results presented here correspond to an aluminum plate which is $5 \mathrm{~mm}$ thick, its size is $500 \times 600 \mathrm{~mm}^{2}$. In the numerical model, the damping of the plate is introduced by a complex Young's modulus $E^{*}=E(1+j \eta)$ with $\eta=10^{-2}$. The acoustic cavity size is $500 \times 600 \times 700 \mathrm{~mm}^{3}$. Damping in the cavity is introduced with a sample of mineral wool, whose acoustic impedance has been characterized in an impedance tube (see fig. 6).

When dealing with experimental reduced models, the choice of the degrees of freedom is of first importance since the model will deeply depend on this choice: the location of the sensors should be carefully choosen in order that the reduced model can represent the behavior of the system on the frequency range of interest. This choice can be done arbitrarily, or based on a non validated model, as shown here.

A finite element model of the coupled system is used to perform this analysis. The values of the 12 first eigenfrequencies are given in table 1.

The choice of a $5 \mathrm{~mm}$ thick plate implies some experimental difficulties, in particular excitation of the plate modes by acoustic excitation requires high acoustic levels. But this configuration is interesting since it exhibits three groups of modes with various coupling levels:

- modes 1 and 2 are decoupled, 

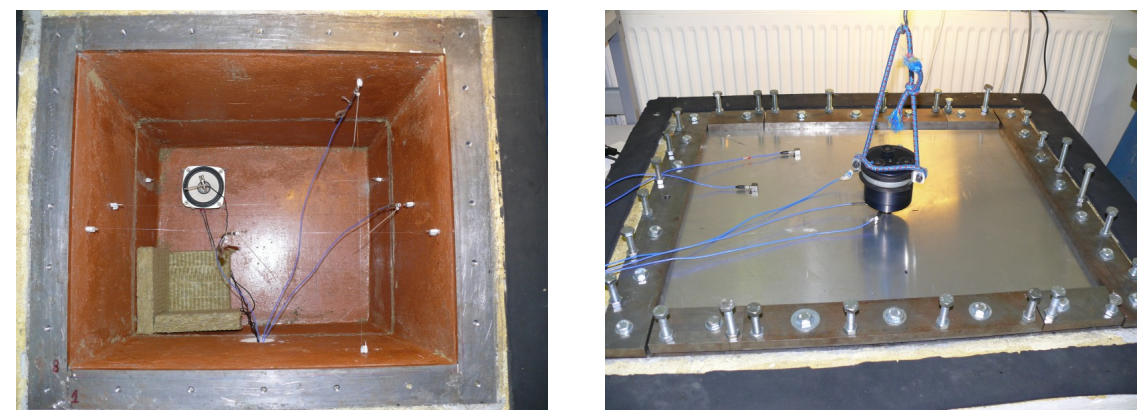

Figure 5: Views of the experimental setup (LVA-INSA Lyon)
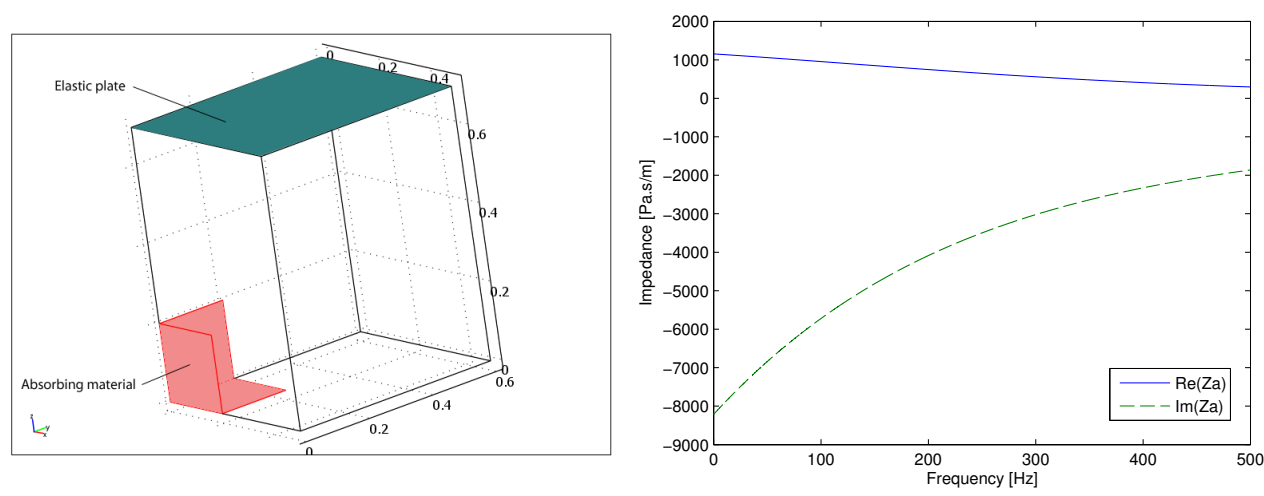

Figure 6: a) Acoustic cavity with absorbing material location, b) Material impedance

\begin{tabular}{|c|c|c|c|c|c|c|}
\hline Order & 1 & 2 & 3 & 4 & 5 & 6 \\
\hline \hline$f(\mathrm{~Hz})$ & 152 & 244 & 271 & 286 & 334 & 347 \\
\hline Nature & $\mathrm{P} 11$ & $\mathrm{C} 001$ & $\mathrm{P} 12$ & $\mathrm{C} 010$ & $\mathrm{P} 21$ & $\mathrm{C} 100$ \\
\hline \hline Order & 7 & 8 & 9 & 10 & 11 & 12 \\
\hline$f(\mathrm{~Hz})$ & 374 & 419 & 440 & 456 & 472 & 487 \\
\hline Nature & $\mathrm{C} 011$ & $\mathrm{C} 101$ & $\mathrm{C} 110$ & $\mathrm{P} 22$ & $\mathrm{P} 13$ & $\mathrm{C} 02$ \\
\hline
\end{tabular}

Table 1: Eigenfrequencies of the system. Dominant movement is indicated by a letter: $\mathrm{C}=$ cavity, $\mathrm{P}=$ plate. Following numbers indicate the mode order in each direction. 
- modes 3 et 4 are weakly coupled,

- modes 5 et 6 are highly coupled.

These different coupling levels are of first interest to investigate the efficiency of the methodology.

\subsection{Optimal pickup design}

We focus on the $[0-350 \mathrm{~Hz}]$ frequency band, in which 6 elastic modes can be observed according to the finite element model. Since in this band there are 3 plate-dominant modes and 3 cavity-dominant modes, it has been decided to use 3 microphones and 3 accelerometers that will constitute the degrees of freedom of the reduced model. The experimental setup is completed by a shaker and a loudspeaker for the excitation of the system.

Several pickup design methodologies are available in structural dynamics literature. In order to find the optimal pickup locations for our system, a QR decomposition of the modal basis has been performed as follow:

- restrain the modal basis to measurable dofs (transverse displacements on the plate, acoustic pressure inside the cavity);

- perform the QR decomposition on both structural and acoustic parts of the modal basis;

- select the degrees of freedom which are identified by the decomposition as pickup locations.

The technique is derived from [39]: if [Ф] is the modal matrix restrained to the measurable degrees of freedom, the QR decomposition allows the determination of the degrees of freedom that participate the most to the independence of vectors:

$$
[\Phi]^{T}[E]=[Q][R],
$$

$[E]$ being a column permutation matrix, $[Q]$ an orthogonal matrix and $[R]$ an upper triangular matrix whose diagonal terms are ranked in descending order.

This technique is known for its discernability and distinguishability performances: a mode is highly discernable if its resonance can be observed on a large number of degrees of freedom, and two modes are distinguishable when their components on measured dofs allow their differentiation.

Without a priori knowledge of the number of sensors to use in each domain, the QR decomposition might be applied on the modal matrix including both structural and acoustic degrees of freedom, but in that case, a preconditionning is mandatory to avoid difficulties associated to the differences of magnitudes in the values of the components. This can be done using the basic approach proposed in section 3 for experimental models, but since a numerical model is available, more advanced techniques are available in literature [40].

Results of the optimal pickup location procedure are given in table 2. Positions $(x, y, z)$ are given in $m$, the cavity dimensions being $0,5 \times 0,6 \times 0,7 \mathrm{~m}^{3}$.

This sensors positioning results in the Auto-MLRMAC matrix shown in figure 7. It compares the value of the matrix when all measurable degrees of freedom are observed, and when only the 6 sensors are used. The reduction of the number of observation points do not deteriorate too much this matrix, which indicates that the sensor locations are pertinent.

The use of this non validated model of the system allows optimal positioning of the sensors before determination of the experimental reduced model. 


\begin{tabular}{|c|c|c|c|}
\hline Sensor ID & $x$ & $y$ & $z$ \\
\hline \hline $\mathrm{a} 1$ & 0,116 & 0,139 & 0,135 \\
\hline $\mathrm{a} 2$ & 0,225 & 0,6 & 0,475 \\
\hline $\mathrm{a} 3$ & 0,284 & 0,234 & 0,622 \\
\hline $\mathrm{s} 4$ & 0,287 & 0,323 & 0,7 \\
\hline s5 & 0,217 & 0,107 & 0,7 \\
\hline s6 & 0,101 & 0,178 & 0,7 \\
\hline
\end{tabular}

Table 2: Locations of the microphones (ID a1 to a3) and accelerometers (s4 to s6)
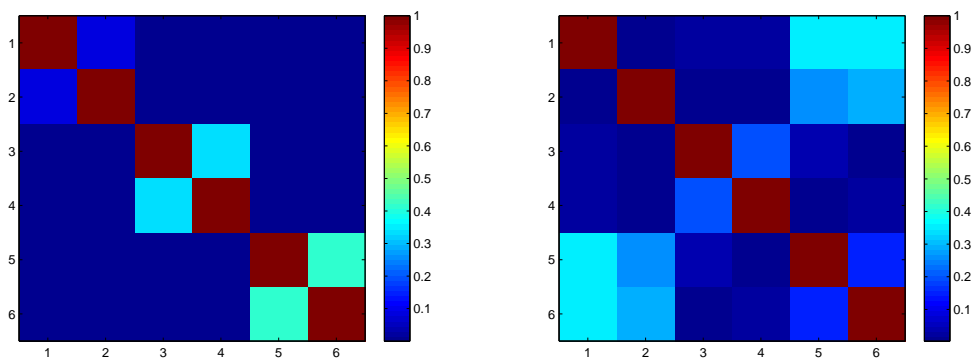

Figure 7: Auto-MLRMAC on the 6 modes of interest, when all measurable dofs are observed (left), and when only 6 dofs are observed (right)

\subsection{Optimal exciter design}

The same methodology, using a QR decomposition, gives optimal exciter location. Table 3 shows the position of the exciters, that correspond to pickup dofs: for experimental reduced model identification, it is mandatory to have the exciters collocated with some measurement points.

\subsection{Reduced model identification from structural excitation}

The easiest way to identify a vibroacoustic reduced model using the inverse procedure presented in section 4 is to use an impedance head at structural excitation point(s) in order to identify the input and cross FRFs. This section intends to show that the methodology provides efficient results when only one structural excitation is used. The efficiency of the methodology is illustrated using FRFs. The figure 8 shows the following curves:

- The Measured FRF. For illustration purpose, only one of the many FRFs is presented here (corresponding to the collocated input and output on the first degree of freedom).

\begin{tabular}{|c|c|c|c|}
\hline Exciter ID & $x$ & $y$ & $z$ \\
\hline \hline 1 & 0,116 & 0,139 & 0,135 \\
\hline 2 & 0,287 & 0,323 & 0,7 \\
\hline
\end{tabular}

Table 3: Locations of loudspeaker (1) and shaker (2) 
- The Modal FRF built from the complex eigenmodes identified using LSCF technique [31]. This is somehow the reference since modes constitute the input before optimal correction.

- The Direct FRF reconstructed using the matrices obtained from eq. (29) from the knowledge of eigenvalues and eigenvectors. This procedure clearly fails in this case, because equations (30) are not strictly verified. Some small differences in the eigendata can induce large discrepancies on the identified system.

- The Proper FRF (referenced as "over") obtained using data with enforcement of the properness condition. In the frequency band of interest, the procedure gives very interesting results: the identified matrices are able to reproduce the behavior of the system.
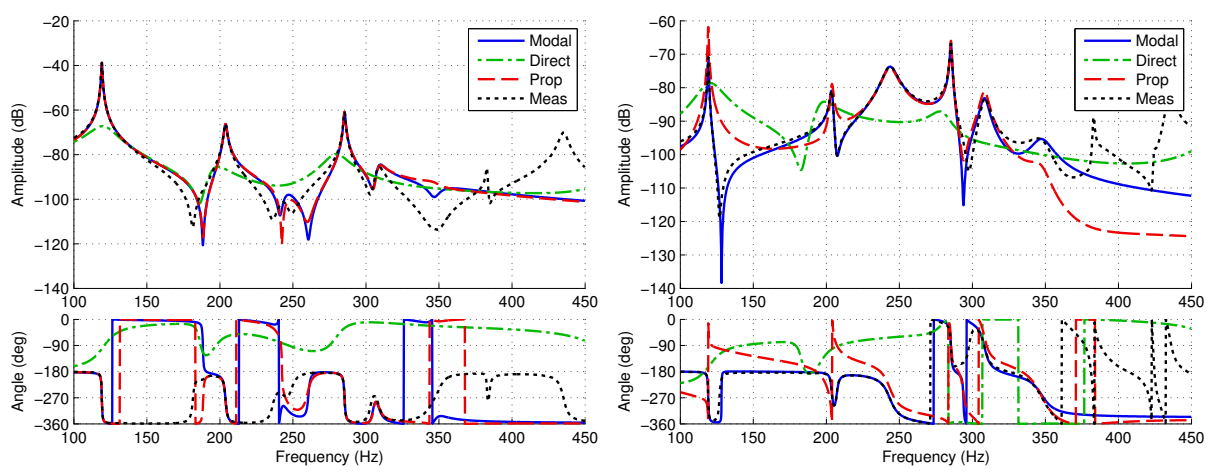

Figure 8: Impact of properness enforcement on reconstructed FRFs (left: FRFs6-s6, right: FRFs6-a1, s=structural, $\mathrm{a}=$ acoustic)

In conclusion, using only structural excitation leads to coherent results. The corresponding reduced model can be applied for any structural excitation in a very efficient way to estimate the vibroacoustic levels. When this model is used with acoustic excitation, the levels are unfortunately badly estimated. The next section will consider the case of acoustic excitation.

\subsection{Vibroacoustic reciprocity}

Only one excitation point is theoretically required to identify the full model. It is nevertheless clear that increasing the number of excitation points will yield to an improvement of the experimental model since new information will be added to improve the conditioning of the inverse problem. In particular, it can be interesting to use acoustic sources. If these sources are monopoles, the vibroacoustic reciprocity relation holds [41, 42, 35]:

$$
\left.\frac{P_{i}}{F_{j}}\right|_{\dot{Q}_{i}=0}=-\left.\frac{\ddot{U}_{j}}{\dot{Q}_{i}}\right|_{F_{j}=0}
$$

This relation must be verified by the structural-acoustic cross FRFs. In particular, it can be useful to identify the acoustic source strength which is not easy to measure directly. As indicated in reference [9], the source can be calibrated in an anechoic chamber using transfer functions between input voltage and structural velocity of the loudspeaker used as acoustic source. Another strategy consists in using reciprocity relationship (52) associated with input voltage measurement 
in order to identify the unknown transfer function between acoustic volume acceleration and input voltage. This is the strategy which has been adopted in this work: figure 9 illustrates the value of the identified transfer function for the experimental setup considered here. Starting from voltage measurement, it has been used to identify the strength of the acoustic source.

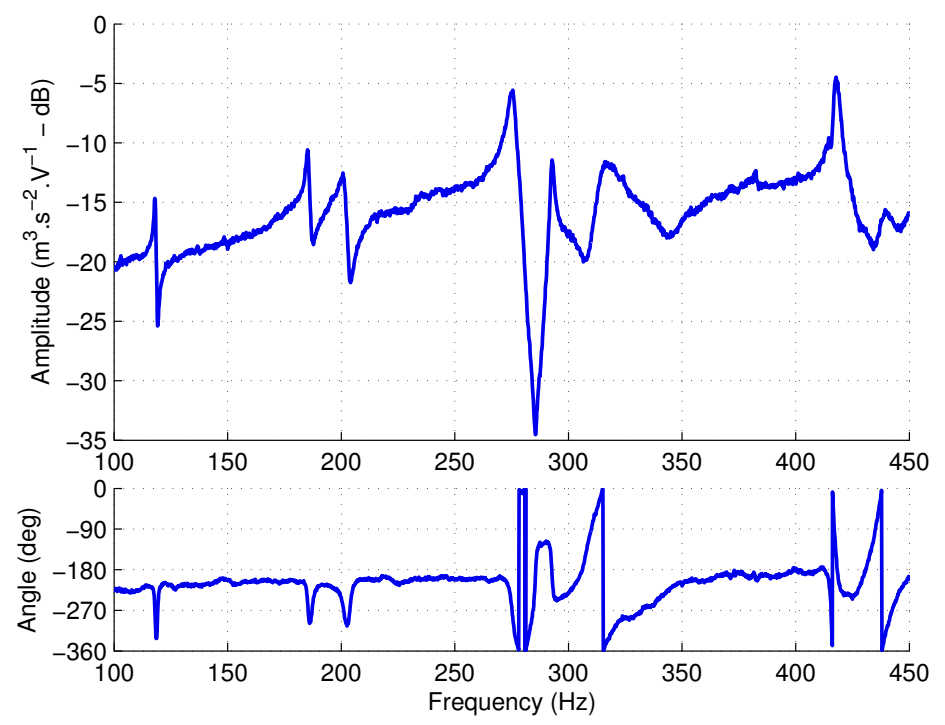

Figure 9: Identification of transfer function between acoustic volume acceleration and input voltage from reciprocity

\subsection{Reduced model identification from acoustic excitation}

In this part, the complex modes identified with acoustic excitation are used to build the reduced model. The results are shown in figure 10. After use of the data obtained from the combination of the measured FRFs with the transfer function shown in figure 9, the LSCF procedure provides the complex modes of the system. As shown in figure 10, the result of the identification is very good on the structure side, while on the cavity side, the synthesized pressure exhibits discrepancies, except at resonances where both curves coincide quite well. Between resonances, it seems that the acoustic source identification procedure is not fully satisfying. This procedure clearly needs more investigation for correct estimation of amplitude on the whole frequency band. However, the topic addressed here being related to the optimal correction of the complex modes, the modal synthesis is used as reference for the correction procedure. Starting from these complex modes, the direct methodology gives completely erroneous results, while the optimally corrected modes allow precise estimation of the matrices of the reduced problem, on both the acoustic and the structure sides.

\section{Conclusions}

In this paper, several tools for model validation in vibroacoustics have been proposed. Damping considerations have been presented to clearly exhibit the validity domain of the approach. 

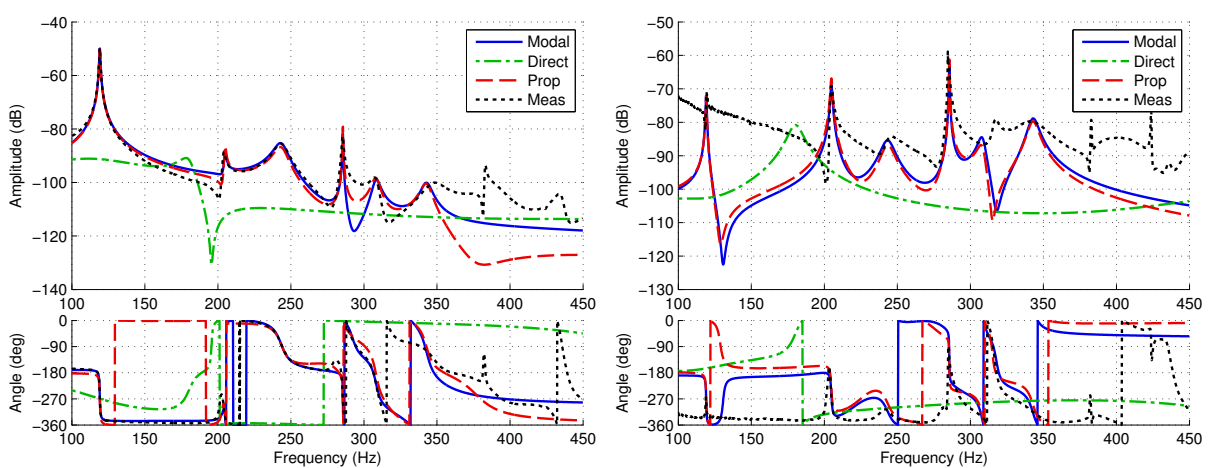

Figure 10: Impact of properness enforcement on reconstructed FRFs (left: FRFa1-s6, right: FRFa1-a1, s=structural, $\mathrm{a}=$ acoustic)

Correlation tools have been derived and an optimal test design strategy has been proposed to define the sensors and actuators locations for vibroacoustic modal analysis. Moreover, a methodology has been provided for the identification of vibroacoustic experimental reduced models, starting from complex modes. These modes are modified in an optimal way to enhance the conditioning of the inverse procedure. The methodology is based either on structural or acoustic excitation. In both cases, levels at resonances are evaluated with a very good degree of confidence. Between resonances, the structural vibrations levels are also very well estimated whatever the excitation is, while for acoustical levels, some investigations are still needed in the case of the use of an acoustic source. The future steps in the developments of the methodology will concern:

- the extension to MIMO (Multi-Input Multi-Output) identification procedure, in order to check the added-value of a combined structural and acoustic excitation;

- the extension of the methodology to frequency-dependent damped configurations, in order to identify reduced models of structures including porous materials for example.

This methodology will allow analysts to perform some hybrid experimental/numerical modelling strategy to build efficient vibroacoustic reduced models. It will also constitute a robust tool to estimate equivalent simple (low-size) physical models of vibroacoustical systems, to properly investigate effects of mass, stiffness or damping changes. In particular, the effect of amplitudedependent damping could be finely analysed with the matrices obtained from the optimally corrected modes.

\section{Acknowledgments}

The authors gratefully acknowledge Céline SANDIER from LVA-INSA Lyon and Charles PEZERAT from LAUM-Le Mans for their contribution to the measurements associated to the experimental part of this work. These measurements have been performed at LVA-INSA Lyon.

The authors acknowledge the financial support of the French National Research Agency (project ANR-12-JS09-008-COVIA). 


\section{References}

[1] L. G. Olson, K. J. Bathe, Analysis of fluid-structure. a direct symmetric coupled formulation based on the fluid velocity potential, Computers \& Structures 21 (1985) 21-32.

[2] G. Everstine, Finite element formulatons of structural acoustics problems, Computers and Structures 65 (3) (1997) 307-321.

[3] J.-F. Deü, W. Larbi, R. Ohayon, Vibration and transient response of structural-acoustic interior coupled systems with dissipative interface, Computer Methods in Applied Mechanics and Engineering 197 (51) (2008) 4894-4905.

[4] W. Desmet, Mid-frequency vibro-acoustic modelling: challenges and potential solutions, in: ISMA, Vol. 2, Leuven, Belgium, 2002, pp. 835-862.

[5] C. Zang, C. Schwingshackl, D. Ewins, Model validation for structural dynamic analysis: an approach to the sandia structural dynamics challenge, Computer Methods in Applied Mechanics and Engineering 197 (29) (2008) 26452659.

[6] M. Imregun, W. Visser, D. Ewins, Finite element model updating using frequency response function data: I. theory and initial investigation, Mechanical Systems and Signal Processing 9 (2) (1995) 187-202.

[7] R. Lin, J. Zhu, Model updating of damped structures using frf data, Mechanical systems and signal processing 20 (8) (2006) 2200-2218.

[8] F. M. Hemez, S. W. Doebling, Review and assessment of model updating for non-linear, transient dynamics, Mechanical Systems and Signal Processing 15 (1) (2001) 45-74.

[9] K. Wyckaert, F. Augusztinovicz, P. Sas, Vibro-acoustical modal analysis: Reciprocity, model symmetry, and model validity, The Journal of the Acoustical Society of America 100 (1996) 3172.

[10] A. Galucio, J.-F. Deü, R. Ohayon, Finite element formulation of viscoelastic sandwich beams using fractional derivative operators, Computational Mechanics 33 (4) (2004) 282-291.

[11] A. Schmidt, L. Gaul, Finite element formulation of viscoelastic constitutive equations using fractional time derivatives, Nonlinear Dynamics 29 (1-4) (2002) 37-55.

[12] J. Allard, N. Atalla, Propagation of Sound in Porous Media: Modelling Sound Absorbing Materials 2e, John Wiley \& Sons, 2009.

[13] O. Dazel, F. Sgard, C.-H. Lamarque, N. Atalla, An extension of complex modes for the resolution of finite-element poroelastic problems, Journal of Sound and Vibration 253 (2) (2002) 421-445.

[14] M. Ouisse, M. Ichchou, S. Chedly, M. Collet, On the sensitivity analysis of porous material models, Journal of Sound and Vibration 331 (24) (2012) 5292-5308.

[15] T. Caughey, M. Okelly, Classical normal modes in damped linear dynamic systems, Journal of Applied Mechanics 32 (3) (1965) 583-588.

[16] S. H. Crandall, The role of damping in vibration theory, Journal of Sound and Vibration 11 (1) (1970) 3-IN1.

[17] C. W. Bert, Material damping: An introductory review of mathematic measures and experimental technique, Journal of Sound and Vibration 29 (2) (1973) 129-153.

[18] L. Gaul, The influence of damping on waves and vibrations, Mechanical systems and signal processing 13 (1) (1999) 1-30.

[19] R. Ohayon, Reduced models for fluid-structure interaction problems, International Journal for Numerical Methods in Engineering 60 (2004) 139-152.

[20] M. Tournour, N. Atalla, Pseudostatic corrections for the forced vibroacoustic response of a structure-cavity system, Journal of the Acoustical Society of America 170 (2000) 2379-2386.

[21] Q. H. Tran, M. Ouisse, N. Bouhaddi, A robust component mode synthesis method for stochastic damped vibroacoustics, Mechanical Systems and Signal Processing 24 (1) (2010) 164-181.

[22] T. Miras, J.-S. Schotté, R. Ohayon, Liquid sloshing damping in an elastic container, Journal of Applied Mechanics 79 (1) (2012) 010902.

[23] M. Prandina, J. E. Mottershead, E. Bonisoli, An assessment of damping identification methods, Journal of Sound and Vibration 323 (3) (2009) 662-676.

[24] A. Srikantha Phani, J. Woodhouse, Viscous damping identification in linear vibration, Journal of Sound and Vibration 303 (3) (2007) 475-500.

[25] A. Srikantha Phani, J. Woodhouse, Experimental identification of viscous damping in linear vibration, Journal of Sound and Vibration 319 (3-5) (2009) 832-849.

[26] D. Bernal, B. Gunes, Extraction of second order system matrices from state space realizations, in: 14th ASCE Engineering Mechanics Conference (EM2000), Austin, Texas, 2000.

[27] S. Ibrahim, A. Sestieri, Existence and normalization of complex modes in post experimental use in modal analysis, in: International Modal Analysis Conference, 13 th, Nashville, TN, 1995, pp. 483-489.

[28] P. Lancaster, U. Prells, Inverse problems for damped vibrating systems, Journal of Sound and Vibration 283 (3-5) (2005) 891-914. 
[29] Q. Zhang, G. Lallement, Comparison of normal eigenmodes calculation methods based on identified complex eigenmodes, Journal of Spacecraft and Rockets 24 (1987) 69-73.

[30] E. Balmès, New results on the identification of normal modes from experimental complex modes, Mechanical Systems and Signal Processing 11 (2) (1997) 229-243.

[31] M. Ouisse, E. Foltête, On the properness condition for modal analysis of non-symmetric second-order systems, Mechanical Systems and Signal Processing 25 (2) (2011) 601-620.

[32] S. Adhikari, J. Woodhouse, Identification of damping: part 1, viscous damping, Journal of Sound and Vibration 243 (1) (2001) 43-61.

[33] S. Adhikari, Optimal complex modes and an index of damping non-proportionality, Mechanical Systems and Signal Processing 18 (1) (2004) 1-28.

[34] S. R. Ibrahim, Computation of normal modes from identified complex modes, AIAA Journal 21 (3) (1983) $446-$ 451.

[35] W. Zhu, J. Liu, Y. Xu, H. Ying, A modal test method using sound pressure transducers based on vibro-acoustic reciprocity, Journal of Sound and Vibration 333 (13) (2014) 27282742.

[36] F. Tisseur, K. Meerbergen, The quadratic eigenvalue problem, SIAM Review 43 (2) (2001) 235-286.

[37] R. Allemang, The modal assurance criterion-twenty years of use and abuse, Sound and Vibration 37 (8) (2003) $14-23$.

[38] K. K. Sairajan, G. S. Aglietti, S. J. Walker, Correlation of finite element models of multi-physics systems, Journal of Sound and Vibration 333 (17) (2014) 4051-4070.

[39] C. Schedlinski, M. Link, An approach to optimal pick-up and exciter placement, in: Proceedings of the SPIE - The International Society for Optical Engineering, 1996, pp. 376-382.

[40] J. Herrmann, M. Maess, L. Gaul, Substructuring including interface reduction for the efficient vibro-acoustic simulation of fluid-filled piping systems, Mechanical systems and signal processing 24 (1) (2010) 153-163.

[41] F. Fahy, Some applications of the reciprocity principle in experimental vibroacoustics, Acoustical Physics 49 (2) (2003) 217-229.

[42] T. Ten Wolde, Reciprocity Measurements in Acoustical and Mechano-Acoustical Systems. Review of Theory and Applications, Acta Acustica united with Acustica 96 (1) (2010) 1-13. 